\title{
Erratum: Experimental determination of the critical spin-glass correlation length in single-crystal CuMn [Phys. Rev. B 102, 064427 (2020)]
}

\author{
G. G. Kenning, D. L. Schlagel, and V. Thompson \\ (Q) (Received 14 August 2021; published 29 September 2021)
}

DOI: 10.1103/PhysRevB.104.099903

Due to a calibration error, the magnetic fields reported in this paper are 3.2 times smaller than the actual magnetic fields. Therefore, in the first section, the reported magnetic field of $5 \mathrm{G}$ is actually $16 \mathrm{G}$, and in the section Magnetic Field Dependence Near $T_{g}$, the magnetic fields now range between 3.2 and 96 G. We submit the corrected Figs. 6-8. This error does not affect our conclusions.

The authors would like to thank Qiang Zhou and Raymond Orbacch for their help in finding this error. 

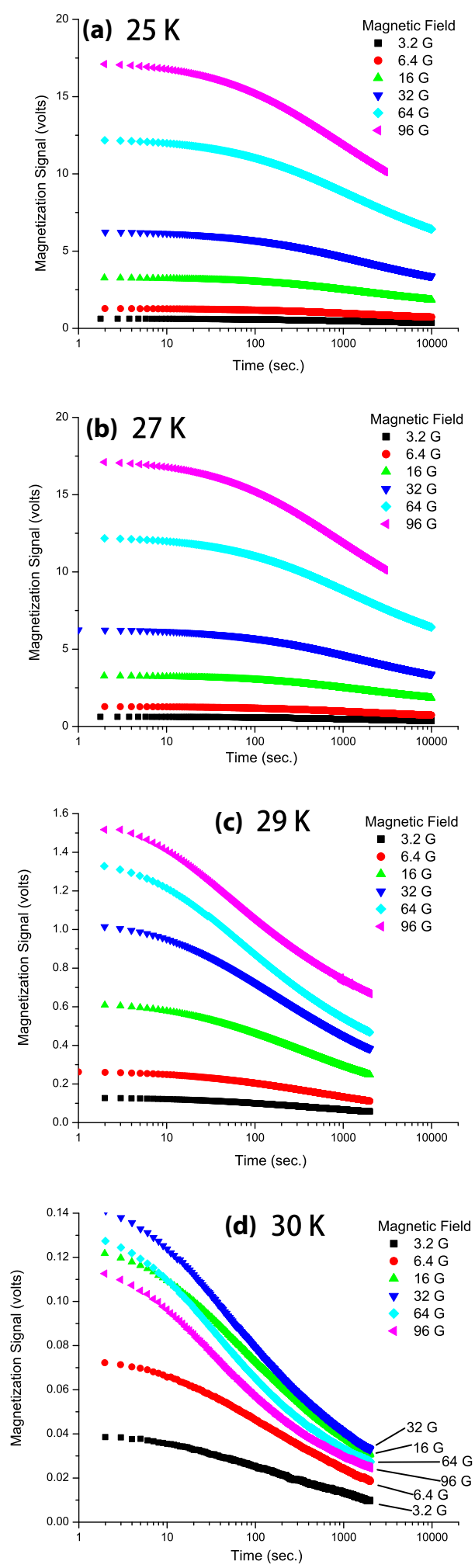

FIG. 6. Variation of TRM decays as a function of magnetic field at temperatures ranging between 25 and $30 \mathrm{~K}$ for a 1000 -s waiting time. Magnetic fields of 3.2, 6.4, 16, 32, 64, and $96 \mathrm{G}$ were measured. 

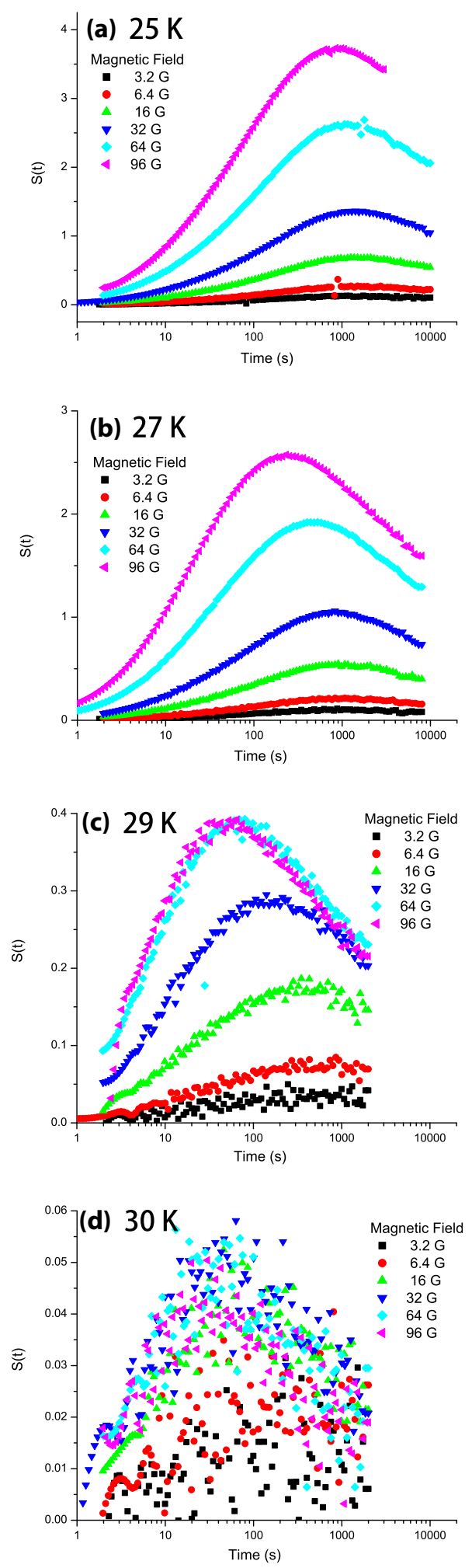

FIG. 7. Variation of $S(t)$ function (data in Fig. 6) as a function of magnetic field at temperatures ranging between 25 and $30 \mathrm{~K}$ for a 1000 -s waiting time. Magnetic fields of 3.2, 6.4, 16, 32, 64, and $96 \mathrm{G}$ were measured. Figure 1(b) displays the same data. The inset in Fig. 1(b) shows the three highest temperature data sets on an expanded scale. 


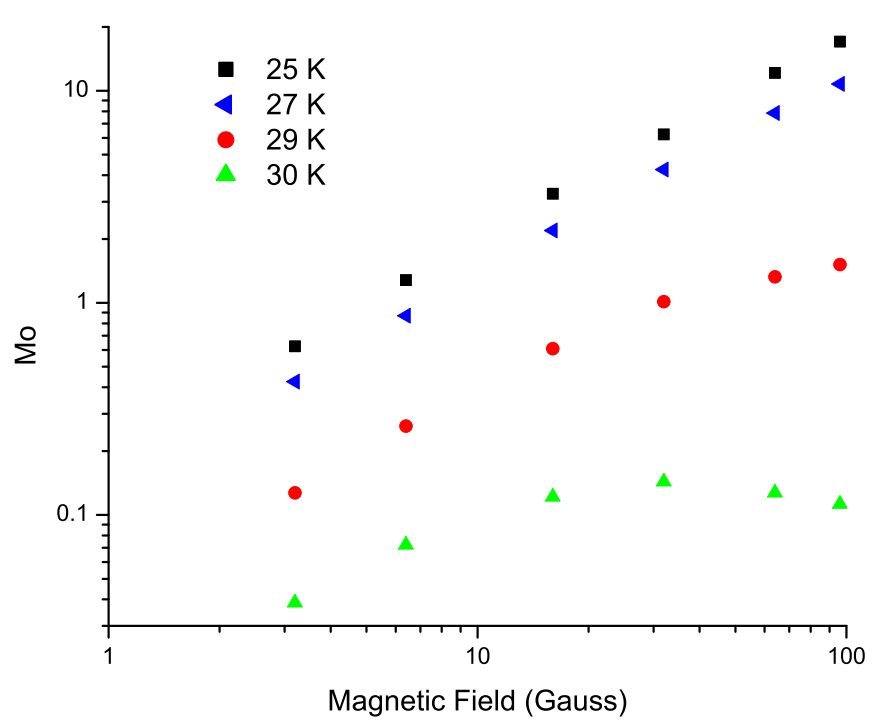

FIG. 8. TRM magnetization measurement at $t=0 \mathrm{~s}$ (solid symbols) for the data in Fig. 6 . 